\title{
Increasing the self-efficacy of individuals with a disability through a theory-based curriculum applied to playing golf
}

\author{
Kiboum Kim ${ }^{1, *}$, David M. Compton ${ }^{1}$ and Gary \\ M. Robb ${ }^{2}$ \\ ${ }^{1}$ Department of Recreation, Park, and Tourism Studies, \\ Indiana University, Bloomington, IN, USA \\ ${ }^{2}$ Former Director, National Center on Accessibility and \\ Former President, National Alliance for Accessible Golf \\ Indiana University, Bloomington, IN, USA
}

\begin{abstract}
Project GAIN (Golf: Accessible and Inclusive Networks) is a theory-based curriculum developed to promote an active life-style and inclusion of individuals with disabilities by enhancing their self-efficacy through golf. Over a 5-year period (2004-2008), 814 participants with and without disabilities from six cities across the USA formally enrolled in Project GAIN. Mentors were used to increase lesson participation, engagement between lessons, and inclusion in golfrelated activities. For this study, data from 327 individuals with disabilities and 295 mentors with and without disabilities were used for analysis purposes. Data included measures of perceived self-efficacy in golf, future plans in golf, and a weekly log of golf-related activities. Paired-samples t-tests and repeated measures ANOVA were utilized to examine the effects of Project GAIN on participants' intention to participate and actual participation in golf-related activities. Results indicated that both study groups (individuals with disabilities and mentors) significantly increased their self-efficacy in golf as well as their intention to play golf in future. Significant increases in golf-related activity were reported in weekly logs over the 5 weeks of data collection. Bonferroni post hoc tests were employed to examine mean differences between weekly observations. Significant mean differences between weeks 1 and 3 , and 1 and 5 were found. The Project GAIN curriculum successfully contributed to improving participants' belief that they could play golf. The study findings support the effectiveness of Project GAIN in increasing golf-related activities that may lead to increased inclusion and physical activity.
\end{abstract}

Keywords: active lifestyle; golf; inclusion; individuals with disabilities; self-efficacy; theory of planned behavior.

\footnotetext{
*Corresponding author: Kiboum Kim, PhD, Assistant Scientist, Indiana University, School of HPER, Department of Recreation, Park and Tourism Studies, 1025 E. Seventh Street, RM 133, Indiana University, Bloomington, IN 47405-7109, USA E-mail:kimki@indiana.edu

Received October 4, 2010; accepted December 8, 2010
}

\section{Introduction}

According to Healthy People 2010, more than half of the US population with disability was physically inactive during discretionary time compared with $36 \%$ of their counterparts without disability (1). Researchers have identified intrapersonal, interpersonal, and structural constraints as inhibitors to participation in physical and social activities (2-6). Interpersonal constraints refer to individual psychological attitudes such as poor self-confidence, unfavorable past experiences, and low expectations. Interpersonal barriers are those that emerge as result of social interaction with others, such as lack of approval or social support. Structural constraints are barriers that arise as a result of conflicts with external conditions such as lack of transportation, shortage of financial resources, environmental inaccessibility, or difficulty in accessing the necessary adaptive equipment (7). In addition to personal and interpersonal factors, perceived physical accessibility may contribute to the level of intention among individuals with disabilities to participate in physical activities such as golf. The Americans with Disabilities Act (ADA) encourages individuals with disabilities to be physically and socially active. Yet, their participation in physical and social activities, including the game of golf, is still constrained by perceived and real accessibility issues (8).

To address some of the constraints identified above, the theory of planned behavior provides explanation of the processes that can affect a person's intention to engage in an active lifestyle (9). The theory suggests that a person's behavior can be affected by behavioral intentions which are influenced by three types of beliefs: (a) the person's attitudes toward targeted behaviors; (b) subjective norms derived from observing the actions of significant others; and (c) the level of perceived behavioral control (9). Specifically, attitude refers to one's belief of how likely it is that the behaviors will result in the anticipated outcome (belief strength), and the degree to which the outcomes are favorable or unfavorable (belief evaluation). Subjective norms refer to one's beliefs of how significant others think that one should perform the given behavior (normative belief), and how motivated the individual is to perform what he thinks he should do (motivation to comply). Finally, perceived behavioral control refers to one's perceived abilities to perform the behavior. It includes one's beliefs about factors that could facilitate or impede one's performance of the behaviors (control belief strength), and how the factors make the performance easier or more difficult when the individual initiates actions to perform the behavior (control belief power). Among these factors, the construct of perceived behavioral control has been accepted as the most important for predicting one's behavioral intention leading to actual participation in the behavior $(9,10)$. 
There has been a general consensus that perceived behavioral control is an extended concept of self-efficacy (11). Bandura posits that self-efficacy is based on a personal belief that one has abilities to perform a behavior under the given circumstance regardless of whether the person has a disability or not (12). Self-efficacy beliefs can be developed and enhanced through four main sources: mastery experience, vicarious experiences, verbal persuasion, and emotional arousal (13). Social scientists have continuously reported the mediating effects of perceived confidence on engagement in physical and recreational activities (13-15). Few researchers, however, have intentionally manipulated self-efficacy conditions and/or planned behavioral control beyond laboratory settings.

In this context, Project GAIN (Golf: Accessible and Inclusive Networks) was developed using the theory of planned behavior and self-efficacy within social cognitive theory. The intent was to document the effects of a community-based model aimed at increasing participation in golf and advancing inclusive opportunities for people with disabilities through the game of golf. This theory-based curriculum departed from a traditional atheoretical, single-clinic approach to teaching golf for individuals with disabilities (16). It was designed to provide instruction in golf, but also to focus on building physical and social behaviors that would lead to ongoing engagement in golf. The formal curriculum was intended to increase golf skills, create a positive attitude toward golf, increase the perceived subjective norm, and enhance perceived behavioral control that results in increased intention to play golf in the future. The curriculum relied on carefully selected mentors to join the individuals with disabilities in the golf lessons, inclusion events and actual golf events (e.g., Champions Challenge, Senior PGA, and Nationwide Tour) to construct ongoing relationships in golf as part of an active physical and social lifestyle (11). Golf professionals and project staff were trained in the application of social cognitive theory within golf lessons using the four main sources of self-efficacy: mastery experiences, vicarious experiences, verbal persuasion, and emotional arousal. Through a complex array of strategies, the curriculum focused on constructing a learning dynamic that would result in planned behavioral intentions to play golf beyond the formal instructional program. Both individuals with disabilities and their mentors received planned curricular golf lessons utilizing self-efficacy and the theory of planned behavior. Activities to increase the intention to play golf following the lessons included planned social activities with mentors, volunteering for professional golf events in the local area, and other golf activities in the community.

The purpose of this study was to examine the impact of a theory-based curriculum on self-efficacy in golfing activities, actual participation, and future intentions to engage in golf-related activities. It was hypothesized that there would be significant mean differences between pretest and posttest in self-efficacy in golf as well as the intentions to play golf in the future. In addition, the hypothesis that there will be significant increases in engagement of golf-related activities over the 5 week period of Project GAIN was tested.

\section{Methods}

\section{Study participants}

Project GAIN participants consisted of two study groups: individuals with disabilities and their mentors. A recruiting process was undertaken in six communities between 2004 and 2008 across the USA (Salt Lake City, UT; Sacramento, CA; Baltimore, MD; Chicago, IL; Toledo, $\mathrm{OH}$; and Howard County, MD) through a variety of media methods (e.g., radio, newspaper, e-mail, direct contact) to enroll participants. When potential subjects expressed an interest in participating in the study, information packets on Project GAIN were distributed. Individuals with disabilities were invited to participate in the project regardless of type and severity of their disability. The majority were individuals with physical disability (i.e., spinal cord injuries, amputees, head trauma injuries, stroke, or other neurological impairments), neuromuscular diseases (i.e., muscular dystrophy or multiple sclerosis), mental retardation. There were 34 categories of disability represented in the pool of individuals with disabilities. All participants 12 years of age or older were allowed to enroll in Project GAIN golf lessons. Assent and consent forms to participate in the research were secured prior to participation. Over the 5 years of the project, 814 individuals and mentors, with and without disability, enrolled in at least one golf lesson offered by Project GAIN. A total of 622 individuals who officially enrolled and completed all survey forms constituted the study population. Data from 327 individuals with disabilities and 295 mentors with and without disabilities were used for analysis purposes. Mentors included family members, friends, volunteers, or professionals willing to attend the lessons and participate with the individual with a disability in the inclusion process (e.g., weekly mentor support, planned social activities, attending golf events) for a period of 3 months. Before participation in the golf lessons, study participants completed a golf skills analysis and selected measurement instruments. Mentors, parents/guardians and experts in cognitive disabilities assisted when necessary.

\section{Measurement}

Three instruments were developed and revised based on existing instruments to measure the impact of Project GAIN $(11,17)$. Selfefficacy in golf (SEG) attempted to determine the level of confidence in preparing for and actually playing the game of golf (12). This instrument was comprised of 31 items in six subscales including: selecting equipment, readiness to play, practicing, playing a round of golf, gathering information about golf, and playing golf with others. Participants responded on a $0-10$-point scale $(0=$ Cannot do at all; 10=Certain I can do). Future plans in golf (FPG) measured the planned intention of participants to participate in golf in the future (17, 18). FPG consisted of three subscales measuring attitude toward golf (three items), subjective norm (two items), and perceived behavioral control (six items). Participants responded using a seven-point Likert scale (1=strongly disagree; $7=$ strongly agree). Finally, a weekly log (WL) was developed to measure the level of engagement in golf-related activities over 5 consecutive weeks. This instrument consisted of seven items measuring active engagement, represented by actually playing golf in some form (e.g., playing nine holes, practicing). An expert review panel was used to assess the content validity for each instrument. Reviewers included: a professor in parks, recreation, and tourism, a doctoral research assistant, PGA golf professional, Project GAIN site coordinators, Project GAIN program evaluator, and a psychometric expert. The project team revised the instruments based on suggestions from the panel. Furthermore, Cronbach's $\alpha$ coefficients 
were calculated to estimate the internal consistency reliability of subscales for each instrument. The estimated coefficients for the SEG ranged from 0.88 to 0.95 and for the FPG from 0.78 to 0.93 , and showed a satisfactory level of internal consistency for items in the scale (19).

\section{Study design}

A repeated measures design with two groups (individuals with disabilities and their mentors) was utilized to examine the effect of the socio-psychological theory-based intervention. Each participant was offered a minimum of five golf instruction sessions over a 3-month period. In addition, they were invited to participate in weekly community-based inclusion activities. The Project GAIN lesson process required that participants complete a certain set of exercises at the end of the lesson, as well as attend and/or view a particular golf activity. Mentors were encouraged to engage in home- and community-based golf-related activities with their mentee. Before and after receiving these treatments, study participants were asked to complete the SEG and FPG questionnaires. Furthermore, participants were asked to indicate within their WL how often they had engaged in each of seven different golf-related activities during the previous week during each of the 5 consecutive weeks of the program.

\section{Data analyses}

The Statistical Package for Social Sciences, Version 19, was used to perform all statistical calculations. A paired-samples t-test was used to compare levels of self-efficacy and future intention to play golfrelated activities before and after participation in the Project GAIN lessons. Data analysis for both groups of individuals with disabilities and their mentors was conducted separately. Differences were considered statistically significant at $\mathrm{p}<0.05$. When a significant difference was observed, a statistical power (Cohen's d) was calculated. In addition, a repeated measures analysis of variance (ANOVA) was applied to identify treatment effects on weekly engagement of golfrelated activities in both groups. Even though there were five data points collected each week during 5 consecutive weeks, three data points (weeks 1, 3, and 5) were utilized in this study owing to a number of missing data in weeks 2 and 4 . The assumption of normality and sphericity was tested before initiating the analysis. No comparison between two study groups was examined since participant type was not considered as an independent variable in this study. Effect size was calculated for each participant group using eta squared $\left(\eta^{2}\right)$. Eta squared values greater than 0.01 are considered small, greater than 0.06 are considered medium, and greater than 0.14 are considered large (19).

\section{Results}

The 622 study participants in Project GAIN (individuals with disabilities $=327$; mentors $=295$ ) were diverse in age (range 1286; mode $=15$ ), disability, and other demographic variables. Of the 295 mentors, 43 participants (14.6\%) who served as mentors were disabled while the other 252 participants were mentors without disability. Approximately $27.5 \%$ of individuals with disabilities were physically disabled, $26.9 \%$ were individuals with mental disorders, and $2.4 \%$ were individuals with visual and hearing loss (missing data $=43.2 \%$ ).
Data for SEG were analyzed using a paired-samples t-test. Results of the analysis indicated that both study groups significantly increased their confidence level in golf from their pretest scores. The SEG mean score for individuals with disabilities increased from 159.41 (pretest) to 201.38 (posttest). The mean difference from the pre to post examination using a paired-samples t-test was $\chi=41.97$. This difference was significant $(\mathrm{p}<0.001)$. Furthermore, the mean scores for the mentor group improved from 249.03 to 273.47 . The mean difference from the pre to post examination using a pairedsamples t-test was significant $(\chi=24.44, \mathrm{p}<0.001)$ (Table 1). These findings suggest that Project GAIN lessons were effective in improving the self-efficacy for golf of both individuals with disabilities and mentors.

Another focus of Project GAIN research was to increase participation in golf-related activities. The normality of the weekly log data was examined before initiating a repeated measures ANOVA test. Several data sets collected from individuals with disabilities (five cases) and their mentors (four cases) were deleted since these individual data were highly skewed. After deleting these nine cases, both skewness and kurtosis of the data distribution appeared to be normally distributed. The original data sets of 147 and 130 were reduced to 142 and 126 for individuals with disabilities and mentor groups, respectively. The null hypothesis of the Sphericity test for data in individuals with disabilities was rejected, while the test for data in the mentor group failed to reject the null hypothesis (19). Results of a repeated measures ANOVA test for individuals with disabilities indicated a significant change in engagement in golf-related activities over time, $F(1.85,260.59)=11.93$, $p<0.001$. The results of Bonferroni post hoc tests indicated that this group appeared to increase their engagement in golf-related activities between weeks 1 and $3(\chi=0.73, p<0.01)$ and between weeks 1 and $5(\chi=1.30, \mathrm{p}<0.001)$. Yet, there was no significant difference between weeks 3 and $5(\chi=0.56, p>0.05)$. The calculated eta squared $\left(\eta^{2}\right)$ value was 0.078 , indicating a medium effect size. The analysis for the mentor group also demonstrated that there was a significant increase in golf-related activity engagement over the 5 weeks of Project GAIN, F(2, 250)=13.97, $\mathrm{p}<0.001, \eta^{2}=0.101$. Again, there were significant differences between weeks 1 and $3(\chi=0.84, p<0.001)$ and between weeks 1 and $5(\chi=1.01, \mathrm{p}<0.001)$. Yet, there was no significant difference between weeks 3 and $5(\chi=0.17, p>0.05)$. Figure 1 depicts a comparison of changes for engagement in golf-related activities between the two study groups. The results of the repeated measure ANOVA test and the Bonferroni post hoc tests are contained in Tables 2 and Table 3, respectively.

The last focus of Project GAIN research was to encourage participants to play golf in the future. The theory of planned

Table 1 Self-efficacy in golf (a paired-samples t-test).

\begin{tabular}{llllll}
\hline Group & $\chi$ & SD & df & t & Cohen's d \\
\hline IWD $^{\mathrm{a}}$ & 41.97 & 69.48 & 291 & $10.32^{\mathrm{b}}$ & 0.60 \\
MNT $^{\mathrm{c}}$ & 24.44 & 41.57 & 260 & $9.50^{\mathrm{b}}$ & 0.59 \\
\hline
\end{tabular}

andividuals with disabilities; ${ }^{\mathrm{b}}$ significance level established at $\mathrm{p}<0.001$; $^{\mathrm{c}}$ mentors. $\chi=$ post test mean - pre test mean. 


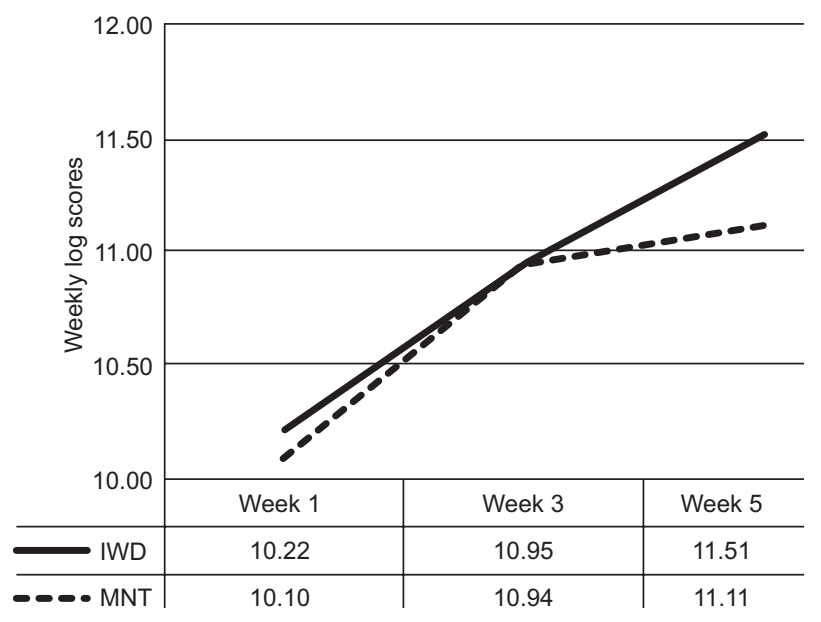

Figure 1 Comparison of changes in weekly engagement of golfrelated activities between two study groups (IWD: individuals with disabilities; MNT: mentors).

behavior suggests that individuals will be more likely to play golf when they increase their intention to do so. FPG was examined for each subscale to determine whether it changed significantly from the pretest to posttest. The differences between pretest and posttest measures for both study groups were calculated using a paired-samples t-test. Results for individuals with disabilities indicated that the posttest measures of both attitude toward golf (ATG) and perceived behavioral control (PBC) increased significantly from their pretest measures, while the measure of subjective norms ( $\mathrm{SN}$ ) did not increase $(\mathrm{p}>0.05)$. The analysis of FPG data for the mentor group indicated that all three subscales increased significantly. Table 4 provides the summary results of the pairedsamples t-test for FPG data.

\section{Discussion}

\section{Self-efficacy in golf}

The results of this analysis suggest that both individuals with disabilities and their mentors improved their self-efficacy. Improving self-confidence was essential to establishing a platform for inclusion, using golf as the vehicle for social change $(12,15)$. The data also suggest that it is important to ensure that the professional instructors adhere to the established protocol provided to ensure that the curriculum intervention could be stable across settings and without variation. While

Table 2 Repeated measures ANOVA for weekly log of participation in golf-related activities.

\begin{tabular}{llrrrll}
\hline Group & Source & \multicolumn{1}{l}{ SS } & \multicolumn{1}{c}{ df } & \multicolumn{1}{c}{ MS } & F & $\eta^{2}$ \\
\hline IWD $^{\mathrm{a}}$ & Weeks & 119.89 & 1.85 & 64.87 & $11.93^{\mathrm{b}}$ & 0.078 \\
& Error & 1417.45 & 260.59 & 5.44 & & \\
MNT $^{\mathrm{c}}$ & Weeks & 73.45 & 2 & 36.78 & $13.97^{\mathrm{b}}$ & 0.101 \\
& Error & 658.44 & 250 & 2.63 & & \\
\hline
\end{tabular}

${ }^{\mathrm{a}}$ Individuals with disabilities; ${ }^{\mathrm{b}}$ significance level established at $\mathrm{p}<0.001$; ${ }^{\mathrm{c}}$ mentors.
Table 3 Comparisons of Weekly Logs between week 1, 3, and 5 (Bonferroni post hoc test).

\begin{tabular}{|c|c|c|c|c|c|}
\hline \multirow[t]{2}{*}{ Group } & \multicolumn{2}{|c|}{ Weeks } & \multirow{2}{*}{$\begin{array}{l}\text { Mean difference } \\
(\mathrm{J}-\mathrm{I})\end{array}$} & \multirow[t]{2}{*}{ SE } & \multirow[t]{2}{*}{$\mathrm{t}$} \\
\hline & $\overline{(I)}$ & $\overline{(\mathrm{J})}$ & & & \\
\hline \multirow[t]{3}{*}{$\overline{\mathrm{IWD}^{\mathrm{a}}}$} & 1 & 3 & 0.73 & 0.23 & $3.17^{b}$ \\
\hline & 1 & 5 & 1.30 & 0.30 & $4.33^{\circ}$ \\
\hline & 3 & 5 & 0.56 & 0.27 & 2.07 \\
\hline \multirow[t]{3}{*}{$\mathrm{MNT}^{\mathrm{d}}$} & 1 & 3 & 0.87 & 0.19 & $4.42^{\circ}$ \\
\hline & 1 & 5 & 1.01 & 0.20 & $5.05^{\mathrm{c}}$ \\
\hline & 3 & 5 & 0.17 & 0.22 & 0.77 \\
\hline
\end{tabular}

andividuals with disabilities; ${ }^{\mathrm{b}}$ significance level established at $\mathrm{p}<0.01$; ${ }^{\mathrm{c}}$ significance level established at $\mathrm{p}<0.001$; ${ }^{\mathrm{d}}$ mentors.

each teaching professional may vary teaching sessions, there are critical pedagogical practices that must be adhered to in using self-efficacy techniques $(12,14)$. For example, using the verbal persuasion technique requires acknowledgement of task accomplishment and attribution to the person rather than chance. The following illustrates the technique: "Andy, you took the club back on the proper line. Well done!"

\section{Weekly activity logs}

Weekly activities logs provided a key indicator of engagement in physical and social activities. In both study groups, there were significant increases in weekly physical activity in weeks 1-3 and 1-5, but no statistical difference in weeks 3-5. Yet, the trends for both groups were positively increased over the 5 week period. Increases in physical activity during weeks 1-3 and 1-5 may be due to several factors. First, the early lessons of weeks 1-3 involved basic elements of the golf swing and stance, while the lessons in weeks 3-5 were comprised of more complex and difficult and self-initiated activities such as playing a few holes or practicing golf. Second, these lessons presented participants with a new activity that many had never previously experienced. In addition, participants' learning activities were customized to their ability level, which may have increased their interest and resulted in task-specific successes, rather than intention to continue playing the game. Finally, in weeks 3-5 there may have been less dialogue or communication between the four sets of individuals involved in Project GAIN lessons (individuals with disabilities, mentors, instructors, and Project GAIN staff). In the future, there

Table 4 Future plan in golf (a paired-samples t-test).

\begin{tabular}{lllrlll}
\hline Group & Subscale & $\chi$ & \multicolumn{1}{l}{ SD } & df & t & Cohen's d \\
\hline IWD $^{\mathrm{a}}$ & ATG & 2.96 & 13.23 & 287 & $3.80^{\mathrm{b}}$ & 0.22 \\
& SN & 0.13 & 3.21 & 290 & 0.68 & - \\
& PBC & 2.80 & 7.26 & 290 & $6.57^{\mathrm{b}}$ & 0.39 \\
MNT $^{\mathrm{c}}$ & ATG & 2.22 & 9.85 & 258 & $3.62^{\mathrm{b}}$ & 0.23 \\
& SN & 0.33 & 2.55 & 259 & $2.09^{\mathrm{d}}$ & 0.13 \\
& PBC & 1.85 & 4.87 & 259 & $6.14^{\mathrm{b}}$ & 0.38 \\
\hline
\end{tabular}

${ }^{a}$ Individuals with disabilities; ${ }^{\mathrm{b}}$ significance level established at $\mathrm{p}<0.001$; ${ }^{\circ}$ mentors; ${ }^{\mathrm{d}}$ significance level established at $\mathrm{p}<0.05 . \chi=$ post test mean - pre test mean. 
will need to be consistent and frequent communication among the groups using various methods, e.g., phone, e-mail, and social media, to ensure the desired outcomes.

\section{Future plans in golf}

While the mean difference in the measure of subjective norm among individuals with disabilities was not significant, the results of this measure indicate that overall both groups of study participants improved their future intention to play golf. These findings suggest that the lessons planned to increase study participants' intentions to play golf in future were effective. However, continuing engagement in golf-related activities by Project GAIN participants was not observed after completion of the study. It is suggested that in future research, data be collected at 1, 3 and 6 month intervals to measure the lasting effects of the lessons on playing golf. As previous studies have indicated, a lack of social support such as social approval is a critical barrier experienced by individuals with disabilities when they initiate participation in recreational activities $(4-8,11,20)$. In addition, there are several limitations in this study that may have influenced future plans to golf, including: the length and frequency of the study intervention; variation in type and severity of disability; lack of mentor training; and other external factors such as negative experiences with golfers without a disability, physical inaccessibility to golf facilities, or difficulties in securing a playing partner. In this context, future studies should pay particular attention to the influence of variation in disabilities and mentor training while controlling for these factors.

\section{Curriculum effectiveness}

From all indications, the Project GAIN curriculum, as originally designed, is pedagogically sound and effective when applied in a group setting. The curriculum is linked to a screening and assessment process that precedes the actual lessons. If the screening and assessment process is carefully executed then the information derived from it leads to a customized learning experience designed for each individual participant. Consistent with the theory of planned behavior, the curriculum was designed to promote socialization from the onset of each lesson, social activities were designed to break down social barriers and reinforce social norms. It appears from verbal comments that were provided by all participants that this segment of the lessons was influential and resulted in self-reported behavior change.

Project GAIN was also based on developing a "partnership" between individuals with disabilities and their mentors, which would lead to playing the game of golf after the formal set of lessons. It appears that the most effective mentors were not those who were closest to the golfer with disability. This implies that the person who is most effective in providing motivational support shares a passion for the game, maintains and sustains the relationship, and is available to assist in addressing such issues as transportation, arranging tee times and assistive devices if required. In several instances, the successful mentor was an individual with a disability, serving as the role model, and having a significant influence on continued engagement. In several cases, the individual with disability returned after a series of lessons to assume the role of mentor to another individual with a disability. More training of the mentors is required in future research to ensure greater effectiveness in relation to inclusion following the initial set of lessons.

\section{Instructional effectiveness}

While it appears that the Project GAIN lessons were a success from the data presented above, there are several observations from the instructional process that are not evident from the data. First, the Professional Golfers' Association and Ladies Professional Golf Association instructors were socialized into the world of disability through exposure to Project GAIN participants. For many, this was their first exposure to individuals with disabilities and, for them, it was impactful and life changing. Second, the instructional program was held primarily at local, public golf courses. This was an excellent introduction for golf course employees to illustrate the potential of individuals with disabilities. Of critical importance was awareness of issues that were essential in creating an atmosphere where physical ability was not the sole factor in enjoying the game of golf.Third, local men's and women's golf associations became aware of the need for the program and appeared to be supportive of individuals with disability playing the game. In some cases, these organizations have become strong advocates for inclusive golf experiences. Four critical actions are required: (a) training of golf professionals and course staff, (b) full compliance with ADA standards, (c) intentional efforts to include golfers with disability in social and playing events, and (d) more applied research to demonstrate the effects of inclusion of individuals with disabilities in golf and related activities that afford physical and social opportunities.

The effectiveness of the Project GAIN instructional program was, to a great extent, based on several factors. We used skilled teaching professionals who could teach effectively in a group setting (10-20 participants). We also had the availability of other teaching and rehabilitation personnel who could address specific issues related to balance, adaptive, or assistive devices necessary to perform basic golf skills, and behavioral, cognitive or communication issues. Project GAIN also ensured the availability of adaptive, assistive, and mobility devices in a sufficient quantity to ensure that those individuals with gripping, balance, stance, vision, or balance issues could be assisted in a timely and effective manner. We used an accommodating venue where the golf course facility staff excelled in customer service and were willing to address individuals with disabilities in a normative manner without fanfare. The project was implemented over a span of time such that the lessons were offered especially during the summer months when vacations may have interfered with the continuity of the lessons. A final fact that contributed to the success was the ability of the model site to provide a "team" of skilled and dedicated staff, technical experts, golf industry personnel, and volunteers to carry out the lessons in an efficient and effective manner. 


\section{Recommendations}

Based on these research findings, it is recommended that in future research and practice, the effectiveness and efficiency of the golf curriculum for both groups (individuals with disabilities and their mentors) could benefit from the following: (a) requiring the teaching professionals (e.g., Professional Golfers' Association and Ladies Professional Golf Association) to complete certification training to ensure adherence with the theory-based program protocols; (b) training all personnel who are involved in the management of the program (screening and assessment, delivery of the lessons and inclusion activities following the lessons); (c) increased scrutiny of the instruments and other essential forms required for the research to ensure that, from the outset, they are complete, accurate, and coded properly; (d) revision and testing of the measurement instruments to ensure that they are reliable and valid; (e) compacting the set of lessons from one 2 hour session each week for 6 weeks to two sessions each week over 3 weeks to reduce attrition; (f) increasing efforts to maintain contact with the participants after the scheduled set of lessons through planned inclusion activities; and (g) providing participants with a "scorecard" of their skill development during the lessons. In addition, there is merit in providing participants with a fitness plan for improving their capacity to engage in daily physical activity, as well as local resources where they can become involved in golf and other activities year round. Finally, there is a need to develop a comprehensive strategy to ensure that more accommodation and inclusiveness is extended to individuals with disabilities and their families by the golf industry, if they are to play golf as a lifelong leisure pursuit. In many cases, golf for individuals with disabilities was not viewed as a common occurrence by the local golf facility staff, but as a rare event or an inconvenience.

It appears that implementation of the Project GAIN model and other theory-based programs could lead to long-term engagement and identity with the game of golf $(10,11,14$, $15)$. There is a need to test theoretically anchored instruction programs that address motor skill development, increased social skills, and the intention to engage in physical activities including golf $(9,15,20)$. Furthermore, model golf programs should be directed at increasing self-confidence through lead-up activities, and an active lifestyle (12). Finally, these programs should not result in periodic agency-sponsored events that isolate individuals with disabilities and their families. Instead, they should lead to efforts that create a seamless, open, and inclusive environment where all individuals, regardless of their ability, can take up and remain in the game of golf $(14,21)$.

\section{Acknowledgments}

This research was supported by a grant to Indiana University, National Center on Accessibility, Department of Recreation, Park and Tourism Studies, School of HPER, Bloomington, Indiana, from the United States Golf Association. Further support was provided by the National Alliance for Accessible Golf, PGA of America, The
PGA Tour, and Ladies Professional Golf Association. Special thanks are extended to the University of Utah for hosting the research team.

\section{Conflict of interest statement}

Authors' conflict of interest disclosure: The authors stated that there are no conflicts of interest regarding the publication of this article. Research support played no role in the study design; in the collection, analysis, and interpretation of data; in the writing of the report; or in the decision to submit the report for publication.

Research funding: United States Golf Association.

Employment or leadership: None declared.

Honorarium: None declared.

\section{References}

1. US Department of Health and Human Services. Objectives for improving health. In: Healthy People 2010. Conference edition. Washington, DC: DHHS, 2000.

2. Crawford DW, Jackson EL, Godbey G. A hierarchical model of leisure constraints. Leis Sci 1991;13:309-20.

3. Buffart LM, van der Ploeg HP, Bauman AE, Van Asbeck FW, Stam HJ, Roebroeck ME, et al. Sports participation in adolescents and young adults with myelomeningocele and its role in total physical activity behaviour and fitness. J Rehabil Med 2008;40:702-8.

4. Rimmer JH, Riley B, Wang E, Rauworth A, Jurkowski J. Physical activity participation among persons with disabilities: barriers and facilitators. Am J Prev Med 2004;26:419-25.

5. Buffart LM, Westendorp T, van den Berg-Emons RJ, Stam HJ, Roebroeck ME. Perceived barriers to and facilitators of physical activity in young adults with childhood-onset physical disabilities. J Rehabil Med 2009;41:881-5.

6. Heller T, Hsieh K, Rimmer J. Barriers and supports for exercise participation among adults with Down syndrome. J Gerontol Soc Work 2002;38:161-78.

7. Pike H, Walker J, Collins J, Hodges J. An investigation of ADA compliance of aquatic facilities in the North Texas area. Am J Health Promot 2008;23:139-46.

8. Barbookles J. Comment: creating reasonable accommodations without an undue burden: the future effects and ADA will have on golf courses. Gold Gate Univ L Rev 2003;33:71-104.

9. Ajzen I. Perceived behavioral control, self-efficacy, locus of control, and the theory of planned behavior. J Appl Soc Psychol 2002;32:665-83.

10. Chiou JS, Huang CY, Chuang MC. Antecedents of Taiwanese adolescents' purchase intention towards the merchandise of a celebrity: the moderating effect of celebrity adoration. J Soc Psychol 2005;145:317-32.

11. Armitage CJ, Conner M. Efficacy of the theory of planned behavior: a meta-analytic review. Br J Soc Psychol 2001;40:471-99.

12. Bandura A. Social learning theory: the exercise of control. New York: Freeman, 1997.

13. White K, Terry D, Troup C, Remple L, Morman P. Predicting the consumption of foods low in saturated fats among people diagnosed with type 2 diabetes and cardiovascular disease. The role of planning in the theory of planned behaviour. Appetite 2010;55:348-54.

14. Peterson JJ, Lowe JB, Peterson AN, Nothwehr FK, Janz KF, Lobas JG. Paths to leisure physical activity among adults with 
intellectual disabilities: self-efficacy and social support. Am J Health Promot 2008;23:35-42.

15. Anderson ES, Wojcik RA, Winett RA, Williams DM. Socialcognitive determinants of physical activity: the influence of social support, self-efficacy, outcome expectations, and self-regulation among participants in a church-based health promotion study. Health Psychol 2006;25:510-20.

16. Drance D, Block ME. Accessible golf: making it a game fore all. Champaign, IL: Human Kinetics, 2005.

17. Theodorakis Y, Bagiatis K, Goudas M. Attitudes toward teaching individuals with disabilities: application of planned behavior theory. Adapt Phys Activ Q 1995;12:151-60.
18. Francis JJ, Eccles MP, Johnston M, Walker A, Grimshaw JM, Foy R, et al. Constructing questionnaires based upon the theory of planned behavior. Newcastle upon Tyne: ReBECI, 2004.

19. Cohen J. Statistical power analysis for the behavioral sciences, 2nd ed. Hillsdale, NJ: Lawrence Erlbaum, 1998.

20. Weiss J, Diamond T, Demark J, Lovald B. Involvement in special Olympics and its relations to self-concept and actual competency in participants with developmental disabilities. Res Dev Disabil 2003;24:281-305.

21. Santiago M, Coyle C. Leisure-time physical activity and secondary conditions in women with physical disabilities. Disabil Rehabil 2004;26:485-94. 